\title{
A Fault Diagnosis Scheme for Rolling Bearing Based on Particle Swarm Optimization in Variational Mode Decomposition
}

\author{
Cancan Yi, Yong Lv, and Zhang Dang \\ School of Mechanical Engineering, Wuhan University of Science and Technology, Wuhan 430081, China \\ Correspondence should be addressed to Yong Lv; lvyong@wust.edu.cn
}

Received 20 January 2016; Revised 15 May 2016; Accepted 23 May 2016

Academic Editor: Athanasios Chasalevris

Copyright (c) 2016 Cancan Yi et al. This is an open access article distributed under the Creative Commons Attribution License, which permits unrestricted use, distribution, and reproduction in any medium, provided the original work is properly cited.

\begin{abstract}
Variational mode decomposition (VMD) is a new method of signal adaptive decomposition. In the VMD framework, the vibration signal is decomposed into multiple mode components by Wiener filtering in Fourier domain, and the center frequency of each mode component is updated as the center of gravity of the mode's power spectrum. Therefore, each decomposed mode is compact around a center pulsation and has a limited bandwidth. In view of the situation that the penalty parameter and the number of components affect the decomposition effect in VMD algorithm, a novel method of fault feature extraction based on the combination of VMD and particle swarm optimization (PSO) algorithm is proposed. In this paper, the numerical simulation and the measured fault signals of the rolling bearing experiment system are analyzed by the proposed method. The results indicate that the proposed method is much more robust to sampling and noise. Additionally, the proposed method has an advantage over the EMD in complicated signal decomposition and can be utilized as a potential method in extracting the faint fault information of rolling bearings compared with the common method of envelope spectrum analysis.
\end{abstract}

\section{Introduction}

Rolling bearing is one of the most commonly used parts in rotating machinery to support rotating shafts. Due to the fact that its health state is directly related to the safety and a stable operation of the machine, the research of rolling bearing fault diagnosis has a great significance in actual application [1]. However, the early incipient fault feature is very faint and interfered by the strong background noise [2]. When the rolling bearing is in failure due to the influence of load, friction, noise, and other factors, the measured vibration signal is a multicomponent amplitude-modulated-frequencymodulated (AM-FM) signal using system's natural frequency as carrier frequency and the fault characteristic frequency as modulation frequency, respectively. Thus, obtaining the AMFM signal by the original signal decomposition and reducing the effect of noise are the emphasized research content in extracting the early faint fault feature of the rolling bearing.

Currently, there are many methods used to fault diagnosis for rolling bearings, but these methods have some inherent limitations. For instance, wavelet transform (WT) [3, 4] analysis is not adaptive, which is restricted by the selection of wavelet basis function and the number of decomposition levels; empirical mode decomposition (EMD) is lack of theoretical basis and there are some problems in its own algorithm such as the phenomenon of model mixing and the end effect [5-7], which has been used to detect the bearing fault [8]. Although the ensemble empirical mode decomposition (EEMD) [9] has some improvement in solving the problem of model mixing, the actual effect is sensitive to the strong background noise. Empirical wavelet transform (EWT) is introduced by Gilles et al. aimed at extracting a series of AMFM signal from the original signal, which largely depends on choosing the boundaries of Fourier spectrum appropriately $[10,11]$. The sparse decomposition theory [12] and manifold learning method [13] are proposed recently. However, the sparse decomposition relies on the design of the redundant atom library and decomposition algorithm, which has an obvious problem of the large amount of calculation. The manifold learning result is also restricted by the parameter selection of delay time and embedded dimension. From another aspect, the spectroscopy and ferrography of used grease are applied to condition monitoring for rolling element bearing [14], which has a limited capacity in detecting the noniron 
particle in lubricating oil and largely depends on the human experience.

Lately, Dragomiretskiy and Zosso proposed a new variational mode decomposition (VMD) [15] method, which was a new advanced multiresolution technique. A series of iterative updating process was used to minimize the constrained variational model; therefore, the vibration signal was decomposed into various modes or intrinsic mode functions (IMFs) using calculus of variation. It overcame the disadvantage of lacking theoretical basis and noise sensitivity of EMD. Moreover, the VMD method could adaptively determine the relevant frequency band and estimated the corresponding model. Based on the above-mentioned advantage, it was applied to the early fault diagnosis of rolling bearing [16, 17] and economics field [18]. From the theory of VMD algorithm, it can be known that the decomposed result of VMD is restricted by the penalty parameter selection and the number of components. In order to extract the faint fault information of the bearing vibration signal effectively, the parameters' selection needs to be optimized. Taking into account the characteristics of fast convergence speed, small setting parameters and easy to implement particle swarm optimization (PSO) algorithm [19, 20], the paper applies it into the parameters optimization of VMD. The optimized two parameters are obtained by the PSO algorithm, which is used to the process of feature extraction for faint fault signal. After the original signal is decomposed by improved VMD method, the optimal component can be identified by the principle of maximum correlated kurtosis [21]. The index of correlated kurtosis has integrated the characteristics of the kurtosis and correlation function, which can represent the changes of shock signal and avoid the problem of overfitting. The merits of the proposed method can be summarized that the parameter selection and decomposition result of VMD algorithm are less affected by human experience. Using this method to analyze the simulated signal and the measured vibration signal from rolling bearing experiment system, the results indicate that the proposed method can accurately extract the early characteristic frequency of faint fault signal of the rolling bearing. The flowchart about the proposed method is shown in Figure 1.

The rest of the paper is organized as follows. In Section 2, the basic ideas of VMD and PSO are introduced. The simulation signal analysis is described in Section 3. The measured signal in rolling bearing experiment system was analyzed in Section 4. The final conclusions are given in Section 5.

\section{Theories}

2.1. Variational Mode Decomposition (VMD) Algorithm. Variational mode decomposition (VMD) is a new method of signal decomposition based on Wiener filtering, one-dimensional Hilbert transform, and heterodyne demodulation proposed lately by Konstantin Dragomiretskiy. Different from EMD, it defines the mode component as amplitude-modulated-frequency-modulated (AM-FM) signals as follows:

$$
u_{k}(t)=A_{k}(t) \cos \left(\phi_{k}(t)\right),
$$

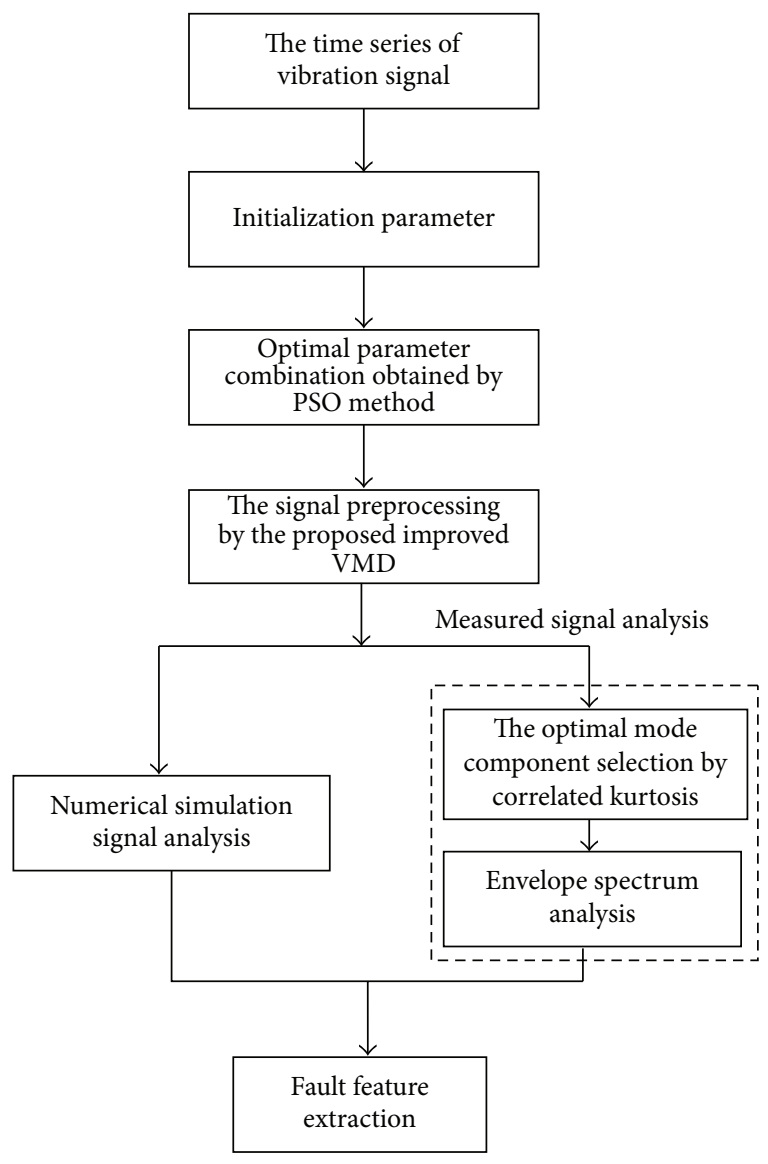

FIgURE 1: The flowchart about the proposed method.

where $\phi_{k}(t)$ is a nondecreasing function; thus $\phi_{k}^{\prime}(t) \geq 0$; the envelope is nonnegative $A_{k}(t) \geq 0$. Additionally, the change of envelope $A_{k}(t)$ and instantaneous frequency $\phi_{k}^{\prime}(t)$ are much slower than $\phi_{k}(t)$. Therefore, the mode component $u_{k}(t)$ can be regarded as a pure harmonic signal with amplitude $A_{k}(t)$ and instantaneous frequency $\phi_{k}^{\prime}(t)$.

The input signal is decomposed by VMD method into the mode component of a specified scale according to the subjective setting scale assuming that each mode is a finite bandwidth signal with a pulse as the center. In order to evaluate the bandwidth of each mode and construct the constraint model of the variational problem, VMD firstly uses the Hilbert transform to obtain the single spectrum of each mode and then transfers them to the fundamental frequency by exponential correction. The bandwidth of each mode is obtained through Gauss smooth demodulation signal finally, which is called as $L^{2}$ norm squared of the gradient. Thereby, the constrained variational problem is given as follows:

$$
\begin{array}{ll}
\min _{u_{k}, \omega_{k}}\left\{\sum_{k}\left\|\partial_{t}\left[\left(\delta(t)+\frac{j}{\pi t}\right) * u_{k}(t)\right] e^{-j \omega_{k} t}\right\|_{2}^{2}\right\} \\
\text { Subject to } \quad \sum_{k} u_{k}=f,
\end{array}
$$

where $f$ is the original signal, $\delta$ is the Dirac distribution, $k$ is number of modes, $\left\{u_{k}\right\}:=\left\{u_{1}, u_{2}, \ldots, u_{k}\right\}$ denotes each 
mode function, $\left\{\omega_{k}\right\}:=\left\{\omega_{1}, \ldots, \omega_{k}\right\}$ indicates each center frequency, $\sum_{k}:=\sum_{k=1}^{k}$ represents the sum of all mode function, and $*$ denotes convolution. Due to the difficulty of solving the constrained problem, the penalty parameter $\alpha$ and the Lagrange multiplication operator $\lambda(t)$ are introduced to convert the above constrained problem to the nonconstraint problem. Thereby obtaining a new solution expression:

$$
\begin{aligned}
& L\left(\left\{u_{k}\right\},\left\{\omega_{k}\right\}, \lambda\right) \\
& :=\alpha \sum_{k}\left\|\partial_{t}\left[\left(\delta(t)+\frac{j}{\pi t}\right) * u_{k}(t)\right] e^{-j \omega_{k} t}\right\|_{2}^{2} \\
& +\left\|f(t)-\sum_{k} u_{k}(t)\right\|_{2}^{2} \\
& +\left\langle\lambda(t), f(t)-\sum_{k} u_{k}(t)\right\rangle .
\end{aligned}
$$

Therefore, the Lagrange multipliers, modal functions, and their corresponding central frequency are iteratively updated by using alternating direction multiplier method (ADMM) to obtain the saddle point in the expression. Specific algorithm of classical VMD is given as the flowing expression.

Step 1. Initialize $\left\{\widehat{u}_{k}^{1}\right\},\left\{\omega_{k}^{1}\right\}, \lambda^{1}, n \leftarrow 0$.

Step 2. The value of $u_{k}, \omega_{k}$, and $\lambda$ is updated according to the following formula:

$$
\begin{aligned}
\widehat{u}_{k}^{n+1}(\omega) & =\frac{\hat{f}(\omega)-\sum_{i \neq k} \widehat{u}_{i}(\omega)+\hat{\lambda}(\omega) / 2}{1+2 \alpha\left(\omega-\omega_{k}\right)^{2}}, \\
\omega_{k}^{n+1} & =\frac{\int_{0}^{\infty} \omega\left|\widehat{u}_{k}(\omega)\right|^{2} d \omega}{\int_{0}^{\infty}\left|\widehat{u}_{k}(\omega)\right|^{2} d \omega}, \\
\lambda^{n+1} & =\lambda^{n}+\tau\left(f-\sum_{k} u_{k}^{n+1}\right) .
\end{aligned}
$$

Step 3. Repeat the iterative process of (2) until the function converges, which is to satisfy the condition of $\sum_{k} \| \widehat{u}_{k}^{n+1}-$ $\widehat{u}_{k}^{n}\left\|_{2}^{2} /\right\| \widehat{u}_{k}^{n} \|_{2}^{2}<e$, where $e$ is a given accuracy requirement.

2.2. Particle Swarm Optimization (PSO) Algorithm. Particle swarm optimization (PSO) is an intelligent algorithm to imitate birds' foraging behavior proposed by Kennedy, through referring to the characteristics of all the individual bird in the process of feeding, which is widely used in solving nonlinear problems.

Each particle in the PSO algorithm is used as the solution of the optimization problem, which has a position and the corresponding speed determined by the optimization function. The algorithm evaluates the pros and cons of all particles by setting the appropriate fitness function. In each iteration, the particles constantly update their speed and position according to the fitness value of individual and the group.
The updated particles continue to search the optimal value in the search space.

Specific configuration steps of PSO are as follows.

Step 1. Establish the appropriate fitness function according to the actual problem. The iteration calculation is carried out by setting the number of iterations, population number, the initial position, and velocity of the particles.

Step 2. Calculate the optimal values $P$ and $G$, respectively, compared with the optimal solution of the current population and retain the better results, where $P$ represents local extremum of individual particles and $G$ indicates global extremum of group particles.

Step 3. Update the position and speed of all particles in the population according to the formula $v_{i d}^{k+1}=\omega v_{i d}^{k}+c_{1} \eta\left(p_{i d}^{k}-\right.$ $\left.x_{i d}^{k}\right)+c_{2} \eta\left(g_{d}^{k}-x_{i d}^{k}\right), x_{i d}^{k+1}=x_{i d}^{k}+v_{i d}^{k+1}$, where $\omega$ is the inertia weight, $c_{1}, c_{2}$ are the learning factor, $\eta$ is the random number between $0 \sim 1$, and $v_{i d}^{k}, x_{i d}^{k}$ are the speed and position of the particle in the $k$ th iteration of the $d$ dimension.

Step 4. Repeat Steps 2 and 3 until meeting the maximum number of iterations.

2.3. Variational Mode Decomposition Based on Particle Swarm Optimization Algorithm. In the traditional algorithm of variational mode decomposition, the user needs to set the penalty parameter and the number of the components before processing the signal because of the theory limitation. From the theoretical study of VMD, it can be known that the larger penalty parameter indicates the smaller bandwidth of each component decomposed by source signal and vice versa. Similarly, inappropriate setting number of components will also result in some unacceptable mode compositions. Therefore, selecting the appropriate parameter group of the component number and the penalty parameter is the key to accurately extract the fault information.

PSO algorithm is a widely used intelligent optimization algorithm compared with other optimization algorithms such as genetic algorithm and artificial fish algorithm. It is suitable for the optimal selection of parameters in consideration of its simple principle and mechanism, fast convergence speed, and, meanwhile, the good performance of global search. The key part of the PSO algorithm based on the variable mode decomposition is the selection of fitness function. Because of the incorrect settings of the penalty parameter and the number of components, some artifact components will generate, which are independent with the source signal. It is acceptable that the artifact components have less similarity with the source signal. Therefore, the cross-correlation coefficient between the decomposed mode component and the original signal is regarded as an evaluation index, which is defined in the following formula:

$$
C=\frac{\sum_{n=1}^{T}(r(n)-\bar{r})(y(n)-\bar{y})}{\left[\sum_{n=1}^{T}(r(n)-\bar{r})^{2} \sum_{n=1}^{T}(y(n)-\bar{y})^{2}\right]^{1 / 2}},
$$


where $r(n), y(n)$ represent the original signal and the mode component, respectively; $T$ is the data length; $C$ represents the cross-correlation coefficient.

From the above analysis, it can be seen that the crosscorrelation coefficient $C$ may fluctuate under the condition of different parameters selection. The largest mean value of cross-correlation coefficient does not imply the best result of mode decomposition. The globally optimal value is achieved by considering the mean value and the variance of crosscorrelation coefficient. The smaller value of variance indicates the less deviation from the mean value. Therefore, the optimal penalty parameters and the number of components can be well obtained by regarding the maximum ratio between the mean value of $C$ and the variance as fitness function. The detailed fitness function is expressed in the following formula:

$$
\text { fit_fun }=\frac{\operatorname{mean}(C)}{\operatorname{var}(C)} .
$$

On the basis of the above theory analysis, VMD based on PSO algorithm is applied to the analysis of simulated signal and the fault feature extraction of rolling bearing experiment system to verify the validity of the method in fault diagnosis.

\section{The Analysis of Simulated Signal}

The measured rolling bearing vibration signal is always consisted of the amplitude-modulated-frequency-modulated (AM-FM) signals, harmonic signal, and noisy signal in actual application. In order to verify the validity of the VMD based on PSO, the fault signal model is built by the following simulated signal:

$$
\begin{aligned}
& x_{1}=\sin \left(2 \pi f_{1} t\right), \\
& x_{2}=\cos \left(2 \pi f_{2} t\right), \\
& x_{3}=\sin \left(2 \pi f_{3} t+\cos \left(2 \pi f_{4} t\right)\right)
\end{aligned}
$$

and then

$$
s=x_{1}+x_{2}+x_{3},
$$

where the frequencies of $f_{1}, f_{2}, f_{3}$, and $f_{4}$ are chosen as $90 \mathrm{~Hz}, 150 \mathrm{~Hz}, 500 \mathrm{~Hz}$, and $270 \mathrm{~Hz}$, respectively. The synthetic signal $s$ is composed by sinusoidal signal $x_{1}$, cosine signal $x_{2}$, and frequency-modulated (FM) signal $x_{3}$. The sampling frequency is set as $1000 \mathrm{~Hz}$ and the sampling point is 1000 . The time-domain graph of simulated signal $s$ is shown in Figure 2.

The VMD based on particle swarm optimization algorithm is applied to decompose the above simulated signal. The number of iterations and the particles is 20 , the inertia weight linear decrease in the iterative process of the initial value is 0.9 , and the final value is 0.4 . The penalty parameter and the number of components optimized and selected by particle swarm optimization (PSO) search algorithm are a collection of $(2064,3)$, and the results of the decomposition are shown in Figure 3.

In the three decomposed components shown in Figures $3(\mathrm{~b}), 3(\mathrm{c})$, and $3(\mathrm{~d})$, the blue line is on behalf of the original

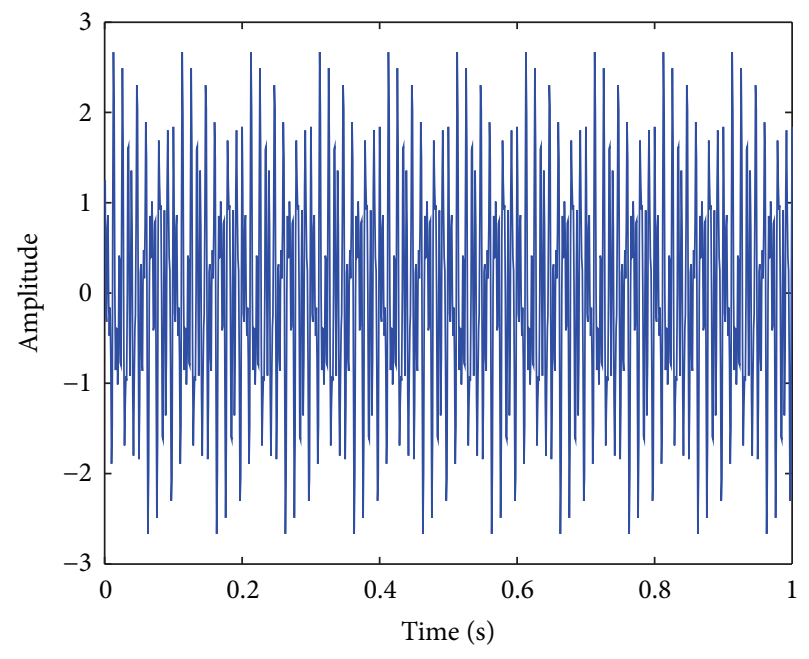

FIGURE 2: The simulated signal $s$.

TABLE 1: The cross-correlation coefficient of reconstruction signal.

\begin{tabular}{lccc}
\hline & Sinusoidal signal & Cosine signal & FM signal \\
\hline $\begin{array}{l}\text { Cross-correlation } \\
\text { coefficient }\end{array}$ & 0.9962 & 0.9985 & 0.9974 \\
\hline
\end{tabular}

TABLE 2: The result of EMD method.

\begin{tabular}{lccc}
\hline & Sinusoidal signal & Cosine signal & FM signal \\
\hline IMF1 & 0.2021 & 0.6132 & 0.5921 \\
IMF2 & 0.7983 & 0.2180 & 0.0032 \\
IMF3 & 0.0276 & 0.0014 & 0 \\
\hline
\end{tabular}

signal and the red line represents the signal after the decomposition. In order to more directly express the result of decomposition, the similarity analysis is carried out between the decomposed component and the original signal by the index of cross-correlation coefficient. Table 1 demonstrates that the proposed method has a perfect ability of complicated signal decomposition.

Empirical mode decomposition (EMD) is used to decompose the above simulated signals, and the results of EMD decomposition are shown in Figure 4. There are eight IMFs decomposed by EMD.

Similarly, the decomposition result also carried out similarity analysis with the original component. Since the similarity of the three signal components (sinusoidal signal, cosine signal, and FM signal) compared with mode components after IMF3 is basically close to zero, so there is only a list of the previous three components shown in Table 2.

From the decomposition results of EMD, it can be known that sine, cosine, and FM signal are partly mixed in IMF1 and IMF2, which cannot be separated well. Compared with the proposed method in this paper, the decomposition effect of VMD is obviously better than EMD, which can well separate the components from the original signal.

The above experimental results show that the proposed method can almost completely separate the components 


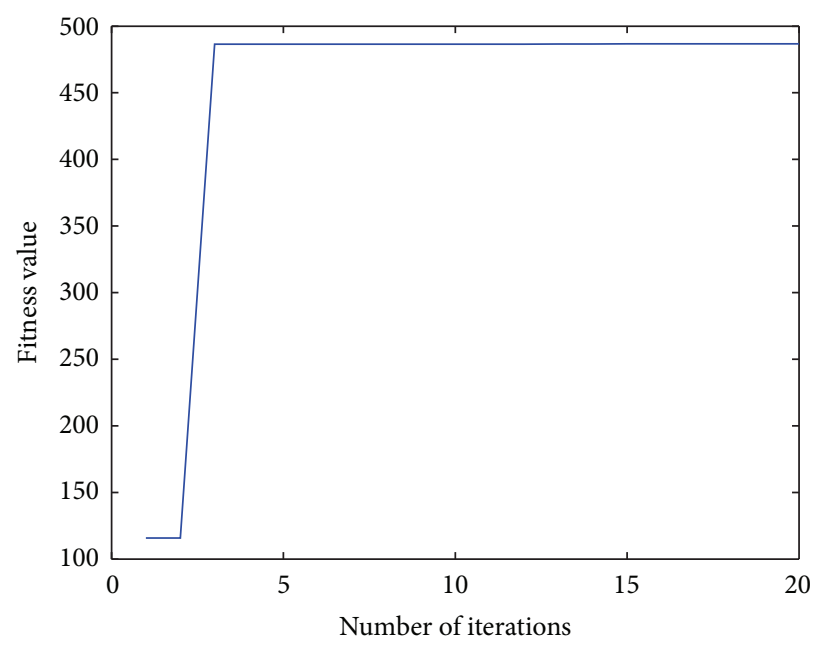

(a) The fitness value during the iteration process

Original cosine signal
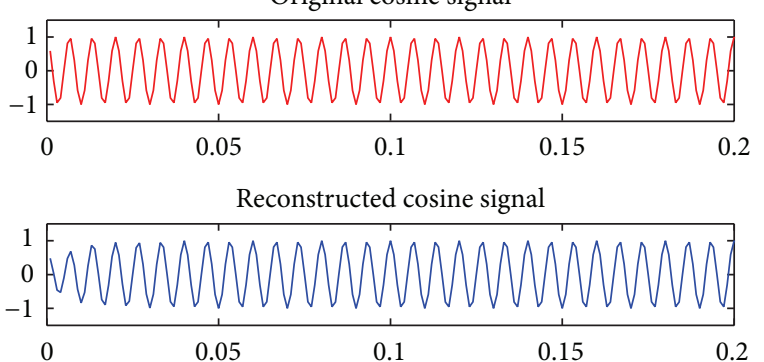

(c) The original and reconstructed cosine signal

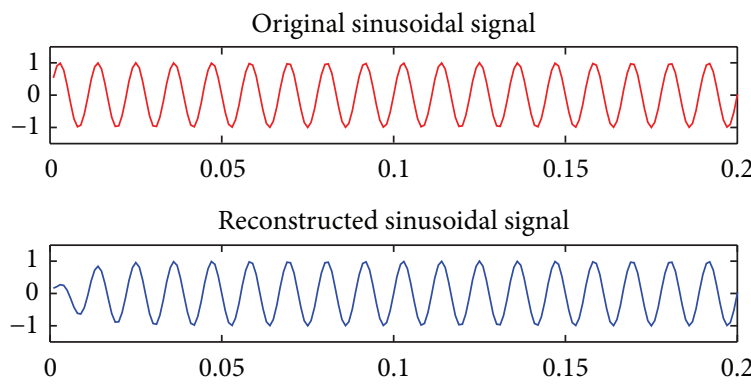

(b) The original and reconstructed sinusoidal signal
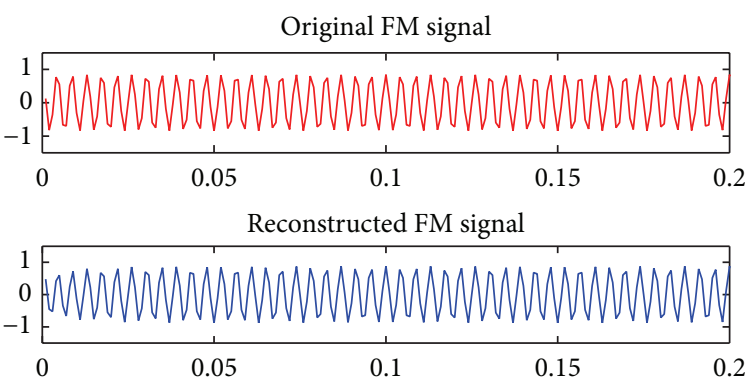

(d) The original and reconstructed FM signal

FIGURE 3: The result of VMD decomposition.

from the simulation signal free of noisy signal. However, the actual measured bearing vibration signal in the runtime is often affected by the strong noise background. Thus, the fault feature information is usually submerged in the noise environment. In order to prove the validity of the method, we discuss the feature extraction effect of this method under the different noise level. In the simulation signal, the Gauss white noise with standard variance being $0.1,0.2,0.4,0.3$, $0.5,0.7$, and 0.9 is added in turn. The cross-correlation coefficient between the decomposed mode components and the original components in different noise conditions is calculated. Results of signal reconstruction by the proposed method are shown in Figure 5.

The noise of different intensity is added in the simulation signal, and then the signal is processed by using the proposed method. From Figure 5, it is obvious that the VMD algorithm based on PSO has a good performance of denoising.

\section{Analysis of Measured Signal in Rolling Bearing Experiment System}

In order to verify that the proposed method is effective in the experiment, the vibration data of rolling bearing experiment system is used to be analyzed. The experimental system is shown in Figure 6. The whole experimental device is driven by a $550 \mathrm{~W}(220 \mathrm{~V} \sim 50 \mathrm{~Hz})$ AC motor. The yellow arrow points the position of the replaceable bearing in Figure 5. In this experiment, the electric spark machining method is used to carry out pitting treatment on the outer ring of replaceable bearing to simulate the faults of the outer ring of bearing. The acceleration signal of the experiment is collected in the vertical direction of the bearing on the right side of the experimental platform using the CSI2130 data analyzer of America. The parameter failure frequencies of fault simulation test-bed are shown in Table 3. It is worth mentioning that the rotating frequency $f_{c}$ and outer fault frequency $f_{o}$ are $24.17 \mathrm{~Hz}$ and $87.01 \mathrm{~Hz}$, respectively.

The time-domain graph of measured bearing fault signal is shown in Figure 7(a). The result of envelope spectrum analysis demonstrates that it is difficult to extract the fault feature due to the interference of noisy signal, which is presented in Figure 7(b).

From Figure 7(b), it is a fact that the 1 to 3 multiplications' frequency of bearing outer fault is interfered by other irrelevant signals such as noisy signal. Particularly, the rotating frequency $f_{c}$ can hard be identification by the means of envelope spectrum analysis. The proposed method in this paper is introduced to optimize and analyze the bearing fault data. In the PSO algorithm, the number of iterations is set as 50 and the population size is selected as 20 . The fitness 

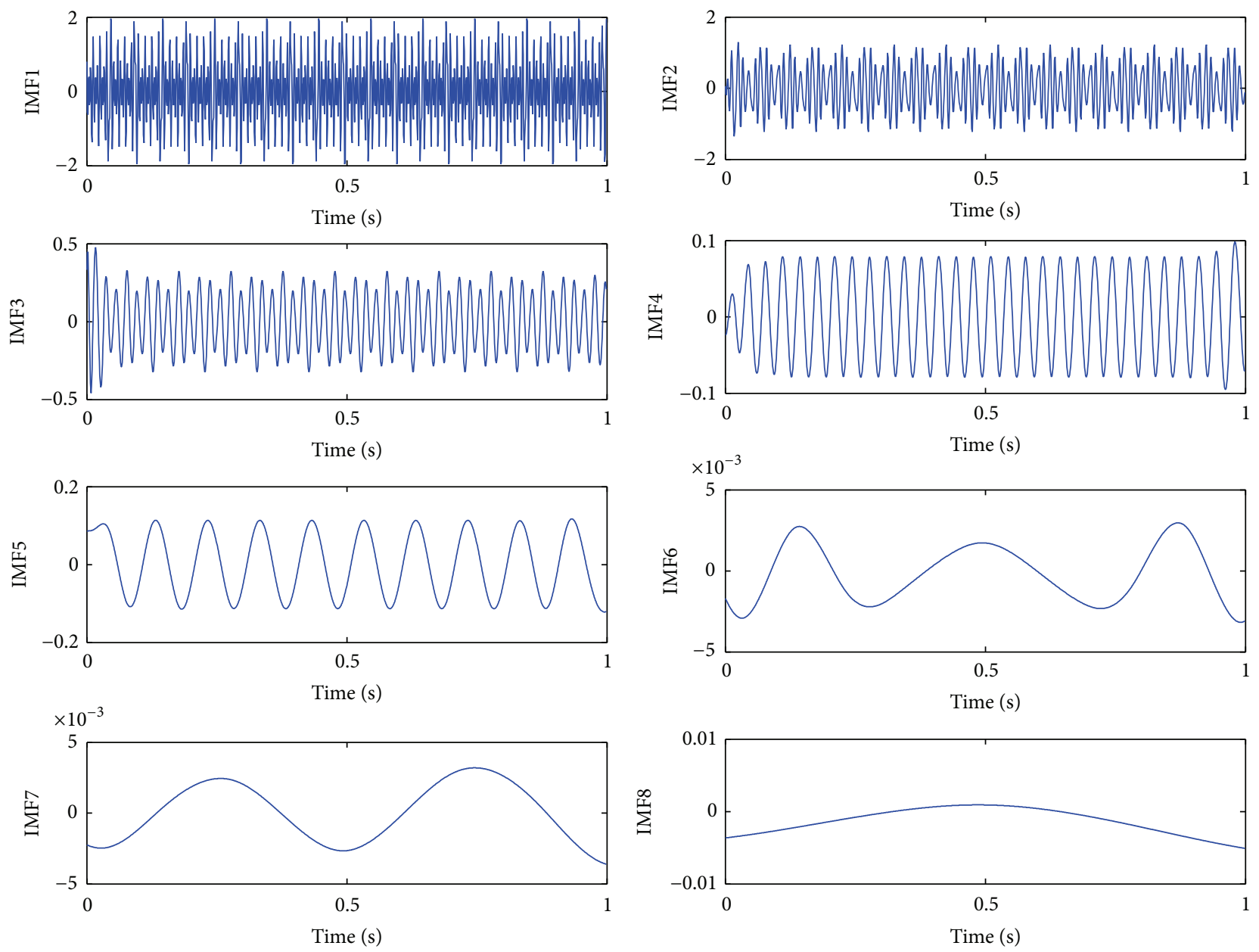

FIGURE 4: The result of EMD decomposition.

TABLE 3: The experimental parameters and fault frequency.

\begin{tabular}{lcccc}
\hline Rotating speed $r / \mathrm{min}$ & Rotating frequency/Hz & Sampling frequency $/ \mathrm{Hz}$ & Sampling time/s & Outer fault frequency/Hz \\
\hline 1450 & 24.17 & 16384 & 1 & 87.01 \\
\hline
\end{tabular}

value during the iteration process is shown in Figure 8. The optimized parameters of the penalty parameter and the number of the components about VMD method are selected as a collection $(1800,10)$. By using the optimized parameters, the bearing fault data are decomposed into ten-mode component and the selection of optimal mode component is carried out. Subsequently, the best representative decomposition component of eighth decomposed component was employed to verify the validity of the method, which was determined by the criterion of maximum correlated kurtosis. The correlated kurtosis of different component was shown in Figure 9 and we can draw a conclusion that the eighth decomposed component has a lager correlated kurtosis value. Figure 10 indicates the result of envelope spectrum analysis for the eighth decomposed component.

It can be seen from the decomposition results of optimized VMD algorithm that the identification accuracy of fault feature frequency is improved compared with the traditional envelope spectrum analysis shown in Figure 7(b). Only 3 multiplications' frequency of the outer ring fault frequency can be found in the envelope spectrum of the original signal, while after optimization, it can find more than 10 multiplications of the fault frequency and detect the rotating frequency $f_{c}$. Additionally, the characteristic spectral line was obvious and less inferred by other spectral lines, which confirms the validity of the proposed method to the fault feature extraction of rolling bearing. The contrastive analysis of the EMD and the proposed method is also carried out, which indicates that the optimized VMD algorithm has a better ability in fault feature identification shown in Figure 11.

\section{Conclusions}

A novel method of particle swarm optimization in variational mode decomposition method was introduced in faint fault feature extraction of rolling bearing. The main conclusions of 


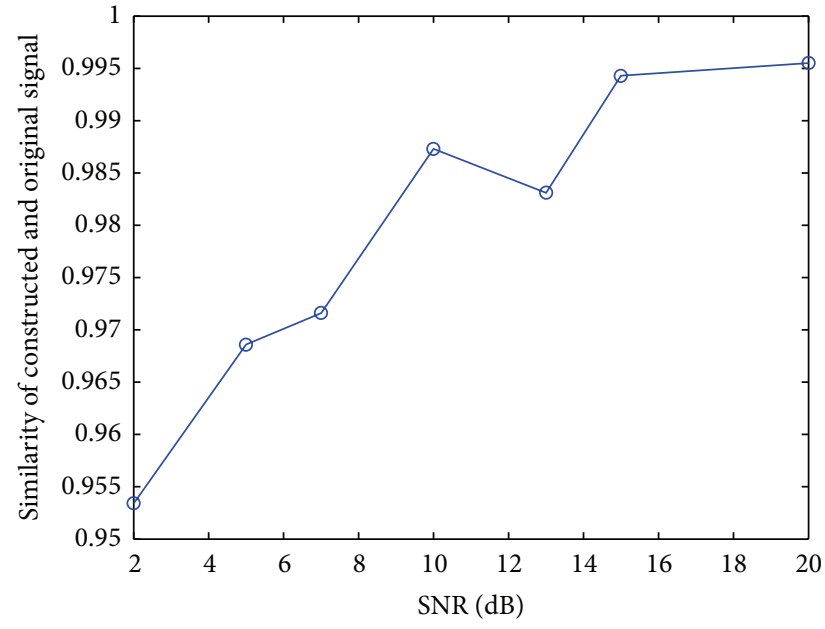

(a) The similarity of sinusoidal component with different noise level

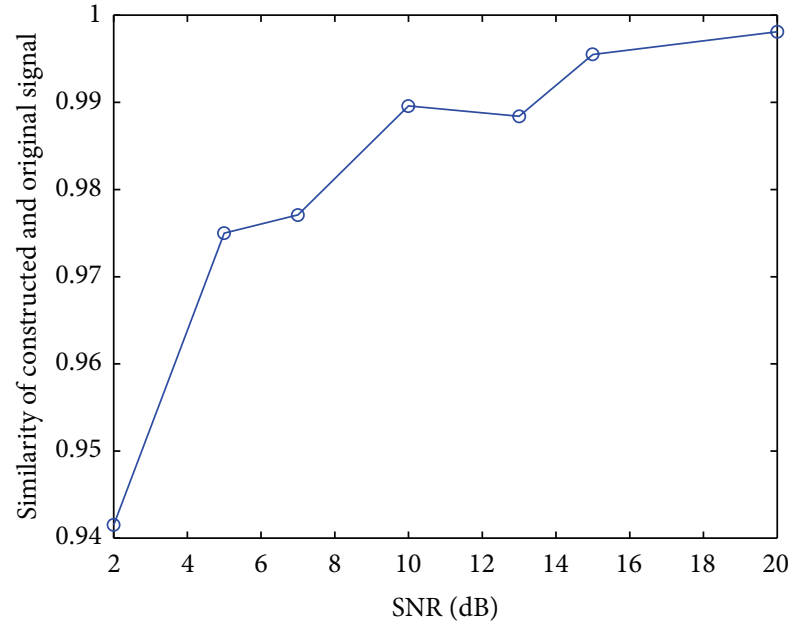

(b) The similarity of cosine component with different noise level

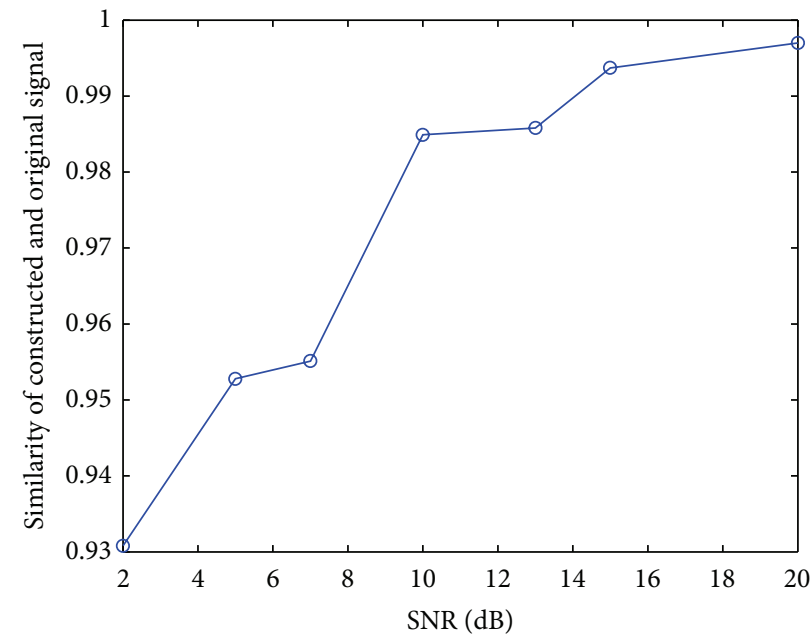

(c) The similarity of FM component with different noise level

FIGURE 5: The results of VMD decomposition with varied noise level.

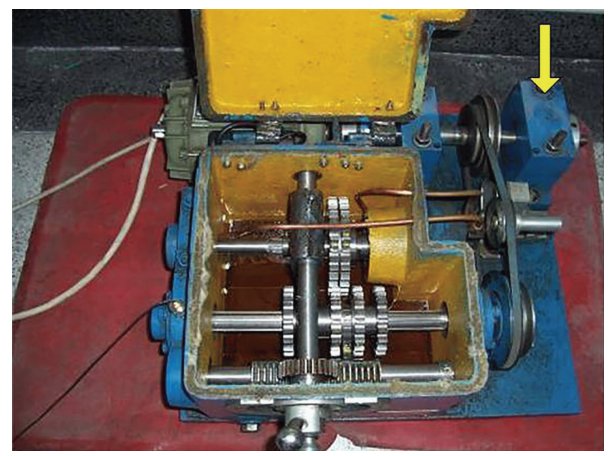

FIGURE 6: The rolling bearing experiment system.

this paper include the following. (1) The particle swarm optimization algorithm was applied to the parameter selection of the optimal penalty parameter and the number of components, which largely depends on the suitable fitness function determined by the maximum ratio between the mean value and the variance of cross-correlation coefficient. Moreover, the maximum correlated kurtosis is used to select the optimal component. It is significant that the proposed method can avoid the interference of human experience and the diagnostic results are more reasonable. (2) Simulated 


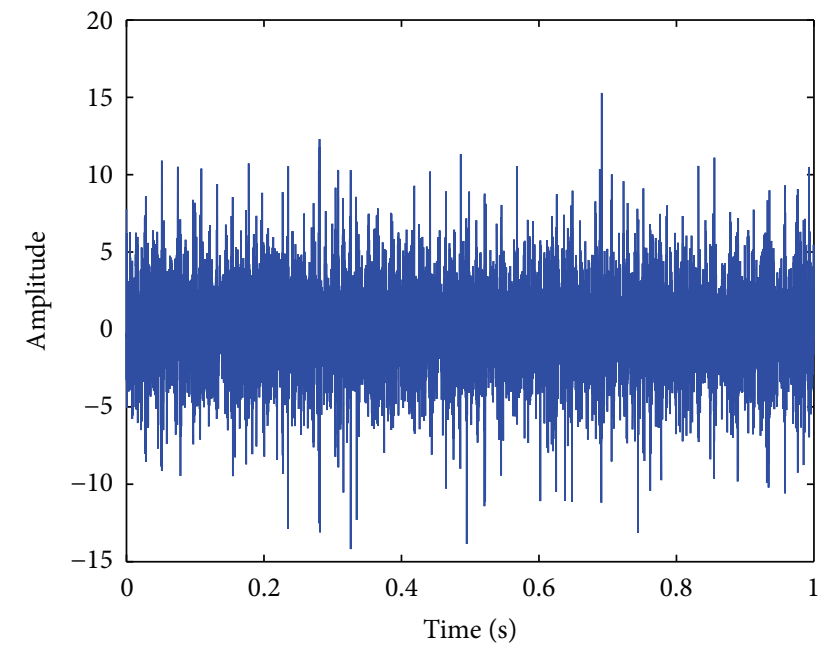

(a) Time-domain graph of the measured signal

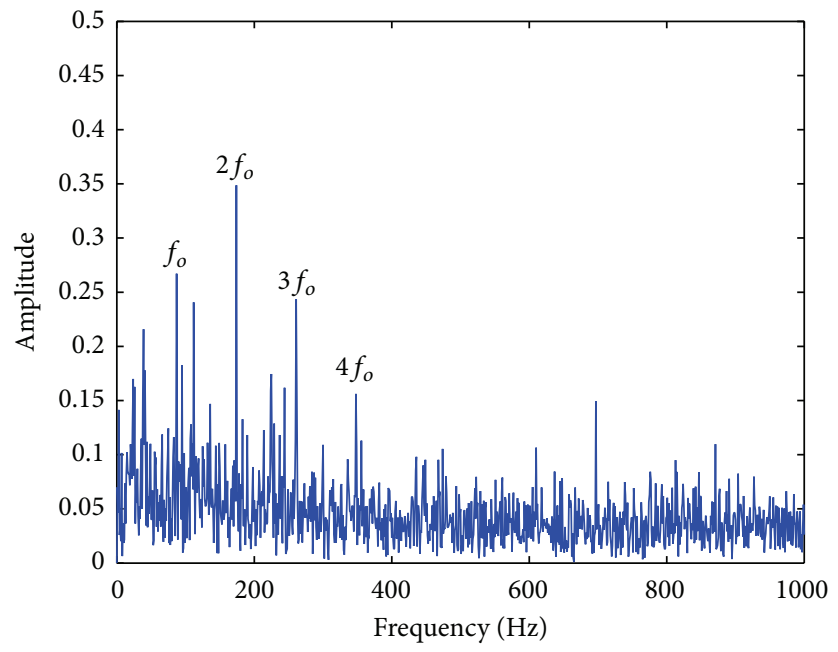

(b) Envelope spectrum graph of the measured signal

Figure 7: The time-frequency diagram of the measured signal.

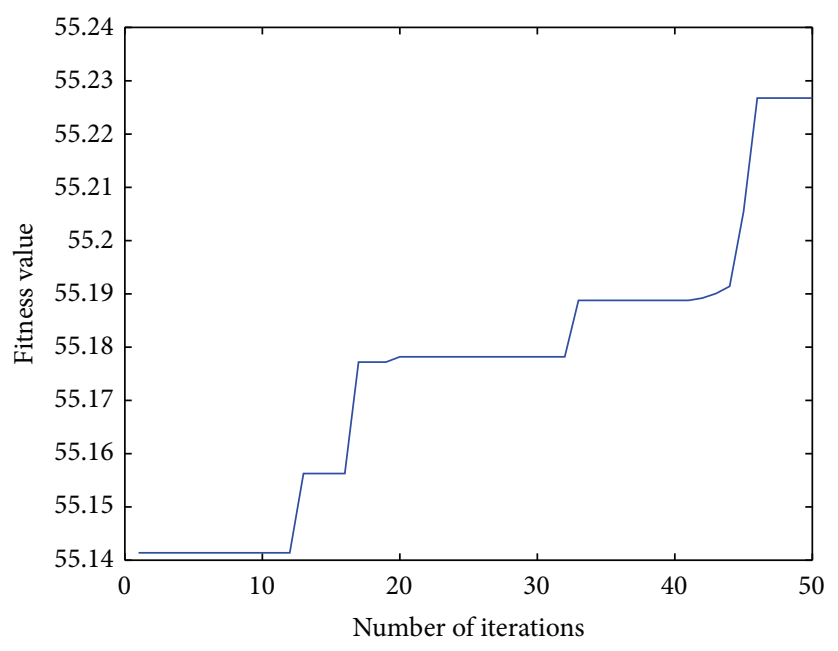

FIgURE 8: The fitness value during the iteration process.

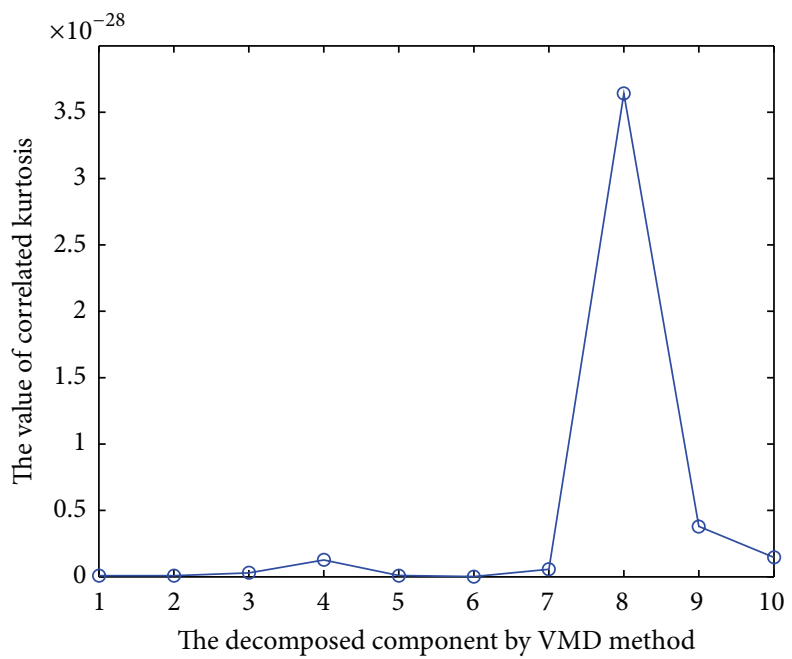

FIgURE 9: The correlated kurtosis of different component. 


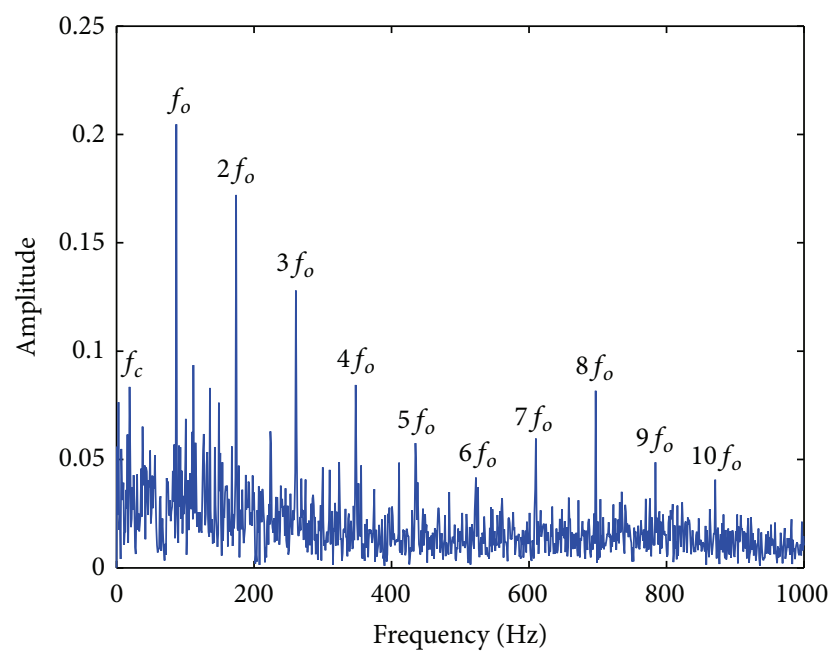

Figure 10: The envelope spectrum analysis of the eighth decomposed component.

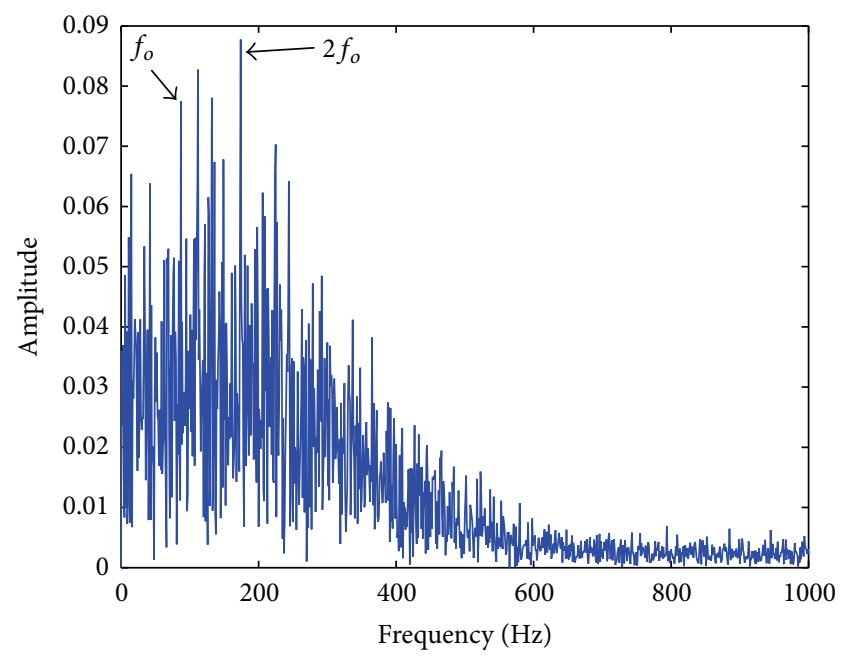

FIgURE 11: The decomposition results of EMD.

signal and measured fault bearing signal measured from the rolling bearing experiment system were used to verify the validity of the method. The result demonstrated that the proposed method has an advantage over the traditional EMD method and envelope spectrum analysis in faint fault signal processing for rolling bearings, which make it possible for the proposed method to be a powerful tool in solving the problem of signal channel bind source separation.

\section{Competing Interests}

The authors declare that there are no competing interests regarding the publication of this paper.

\section{Acknowledgments}

This work was supported by the National Natural Science Foundation of China (nos. 51475339 and 51405353) and the
Key Laboratory of Metallurgical Equipment and Control of Education Ministry, Wuhan University of Science and Technology (2015B11).

\section{References}

[1] M. Zeng, Y. Yang, J. Zheng, and J. Cheng, "Maximum margin classification based on flexible convex hulls for fault diagnosis of roller bearings," Mechanical Systems and Signal Processing, vol. 66-67, pp. 533-545, 2016.

[2] H. Wang, J. Chen, and G. Dong, "Feature extraction of rolling bearing's early weak fault based on EEMD and tunable Q-factor wavelet transform," Mechanical Systems and Signal Processing, vol. 48, no. 1-2, pp. 103-119, 2014.

[3] X. An, D. Jiang, C. Liu, and M. Zhao, "Wind farm power prediction based on wavelet decomposition and chaotic time series," Expert Systems with Applications, vol. 38, no. 9, pp. 11280-11285, 2011.

[4] H. Li and X. Wang, "Detection of electrocardiogram characteristic points using lifting wavelet transform and Hilbert transform," Transactions of the Institute of Measurement and Control, vol. 35, no. 5, pp. 574-582, 2013.

[5] N. E. Huang, Z. Shen, S. R. Long et al., "The empirical mode decomposition and the Hilbert spectrum for nonlinear and non-stationary time series analysis," Proceedings of The Royal Society A: Mathematical, Physical and Engineering Sciences, vol. 454, no. 1971, pp. 903-995, 1998.

[6] C.-F. Lin, "Chaotic visual cryptosystem using empirical mode decomposition algorithm for clinical EEG signals," Journal of Medical Systems, vol. 40, no. 3, pp. 1-10, 2016.

[7] Y. Lei, J. Lin, Z. He, and M. J. Zuo, "A review on empirical mode decomposition in fault diagnosis of rotating machinery," Mechanical Systems and Signal Processing, vol. 35, no. 1-2, pp. 108-126, 2013.

[8] P. Nikolakopoulos and A. Zavos, "Slew bearings damage detection using hilbert huang transformation and acoustic methods," Tribology in Industry, vol. 37, no. 2, pp. 170-175, 2015.

[9] H. Aied, A. González, and D. Cantero, "Identification of sudden stiffness changes in the acceleration response of a bridge to moving loads using ensemble empirical mode decomposition," Mechanical Systems and Signal Processing, vol. 66-67, pp. 314338, 2016.

[10] J. Gilles, “Empirical wavelet transform," IEEE Transactions on Signal Processing, vol. 61, no. 16, pp. 3999-4010, 2013.

[11] Y. Jiang, H. Zhu, and Z. Li, "A new compound faults detection method for A new compound faults detection method for rolling bearings based on empirical wavelet transform and chaotic oscillatorrolling bearings based on empirical wavelet transform and chaotic oscillator," Chaos, Solitons \& Fractals, 2015.

[12] X. Chen, Z. Du, J. Li, X. Li, and H. Zhang, "Compressed sensing based on dictionary learning for extracting impulse components," Signal Processing, vol. 96, pp. 94-109, 2014.

[13] Q. He and X. Wang, "Time-frequency manifold correlation matching for periodic fault identification in rotating machines," Journal of Sound and Vibration, vol. 332, no. 10, pp. 2611-2626, 2013.

[14] R. Liu, "Condition monitoring of low-speed and heavily loaded rolling element bearing," Industrial Lubrication and Tribology, vol. 59, no. 6, pp. 297-300, 2007. 
[15] K. Dragomiretskiy and D. Zosso, "Variational mode decomposition," IEEE Transactions on Signal Processing, vol. 62, no. 3, pp. 531-544, 2014.

[16] C. Aneesh, S. Kumar, P. Hisham et al., "Performance comparison of variational mode decomposition over empirical wavelet transform for the classification of power quality disturbances using support vector machine," Procedia Computer Science, vol. 46, pp. 372-380, 2015.

[17] Y. Wang, R. Markert, J. Xiang, and W. Zheng, "Research on variational mode decomposition and its application in detecting rub-impact fault of the rotor system," Mechanical Systems and Signal Processing, vol. 60, pp. 243-251, 2015.

[18] S. Lahmiri, "Long memory in international financial markets trends and short movements during 2008 financial crisis based on variational mode decomposition and detrended fluctuation analysis," Physica A: Statistical Mechanics and its Applications, vol. 437, pp. 130-138, 2015.

[19] J. Kennedy, "Particle swarm optimization," in Encyclopedia of Machine Learning, pp. 760-766, Springer US, 2010.

[20] M. Yuwono, Y. Qin, J. Zhou, Y. Guo, B. G. Celler, and S. W. Su, "Automatic bearing fault diagnosis using particle swarm clustering and Hidden Markov Model," Engineering Applications of Artificial Intelligence, vol. 47, pp. 88-100, 2016.

[21] G. L. McDonald, Q. Zhao, and M. J. Zuo, "Maximum correlated Kurtosis deconvolution and application on gear tooth chip fault detection," Mechanical Systems and Signal Processing, vol. 33, no. 1, pp. 237-255, 2012. 


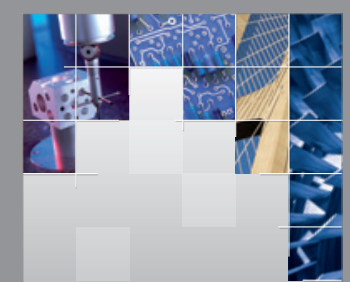

\section{Enfincering}
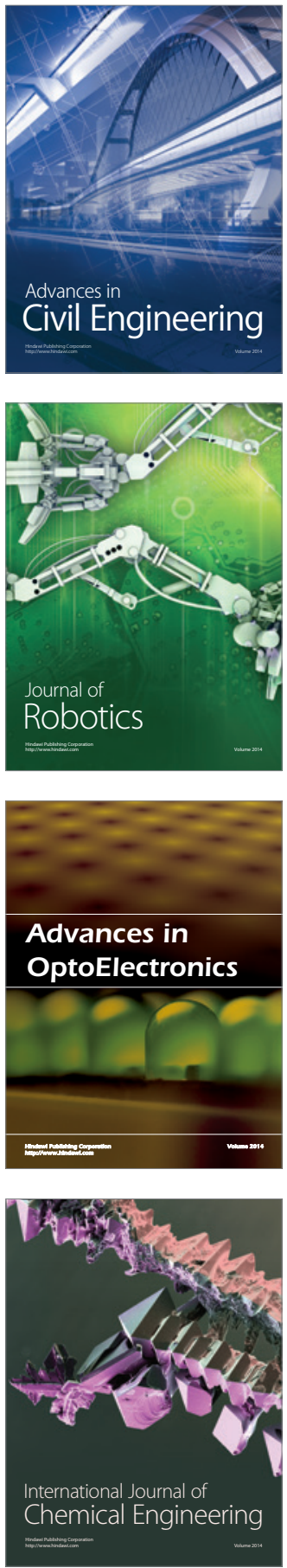

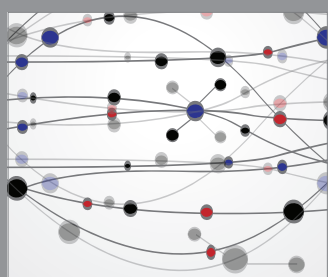

The Scientific World Journal

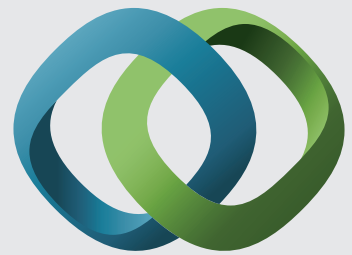

\section{Hindawi}

Submit your manuscripts at

http://www.hindawi.com
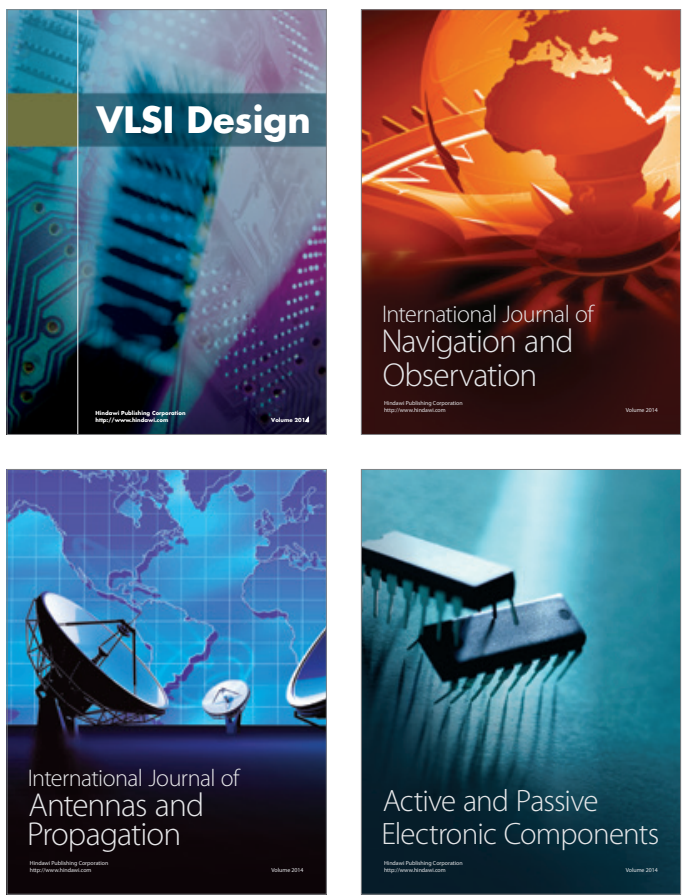
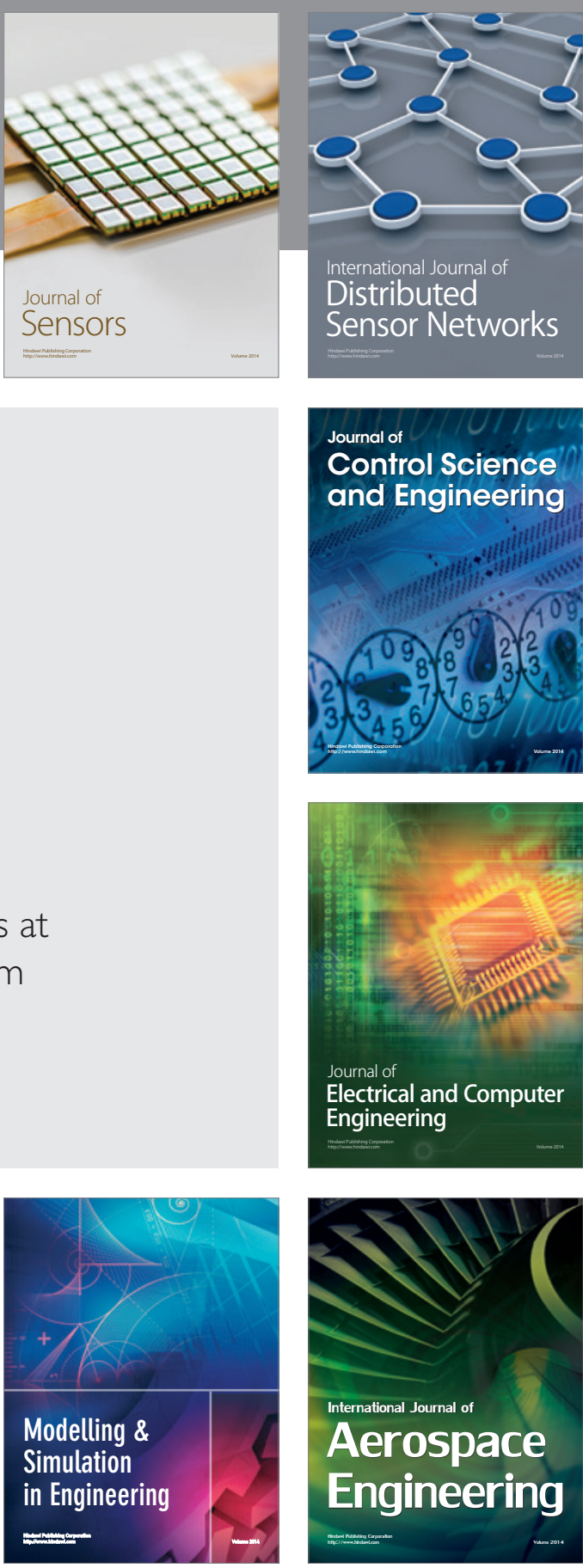

International Journal of

Distributed

Sensor Networks

Journal of

Control Science

and Engineering
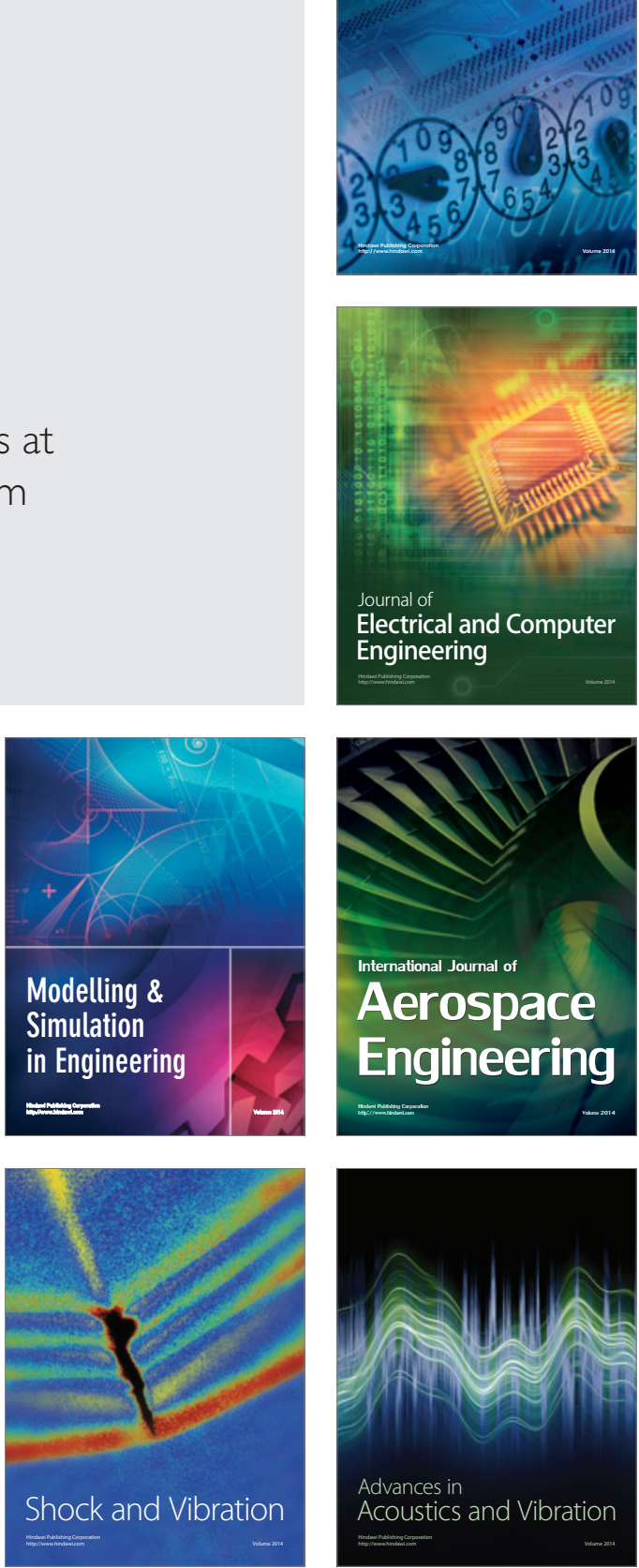